TRANSACTIONS OF THE

AMERICAN MATHEMATICAL SOCIETY

Volume 353, Number 4, Pages 1661-1675

S 0002-9947(00)02673-8

Article electronically published on November 29, 2000

\title{
A SIMILARITY PRINCIPLE FOR COMPLEX VECTOR FIELDS AND APPLICATIONS
}

\author{
S. BERHANU, J. HOUNIE, AND P. SANTIAGO
}

\begin{abstract}
This paper establishes a similarity principle for a class of nonelliptic, smooth complex vector fields in the plane. This principle is used to prove a uniqueness result for a nonlinear Cauchy problem.
\end{abstract}

\section{INTRODUCTION}

In this article we study first-order equations of the form

$$
L w=A w+B \bar{w}
$$

where $L$ belongs to a class of smooth, complex vector fields in the plane (see section 2 ) while $A$ and $B$ are bounded functions. Equation (1.1) is motivated by the classical elliptic equation

$$
\frac{\partial w}{\partial \bar{z}}=A w+B \bar{w}
$$

which had been the subject of many works (see for example, [2], 3], [4], [5], [1] and [16]). In the literature solutions of (1.2) are called pseudoanalytic functions or generalized analytic functions. Pseudoanalytic functions share many properties with analytic functions of a single complex variable. These properties follow from the Similarity Principle which is valid for solutions of (1.2). This principle says that every continuous solution $w$ of (1.2) has the form

$$
w=e^{g} h,
$$

for some holomorphic function $h$ and Hölder continuous $g$. Thus $w$ and $h$ are "similar" in the sense that both $\frac{w}{h}$ and $\frac{h}{w}$ are bounded away from zero on compact sets.

The Similarity Principle holds for any elliptic vector field $L$, since in appropriate coordinates, $L$ becomes a multiple of $\frac{\partial}{\partial \bar{z}}$.

Received by the editors October 14, 1998 and, in revised form, October 25, 1999.

1991 Mathematics Subject Classification. Primary 35F05, 35N10, 35A05; Secondary 35F20, $32 \mathrm{~F} 40$.

Key words and phrases. Similarity principle, locally solvable vector field.

The first author thanks IMPA of Brazil for an invitation to a pde workshop in July, 1997, that facilitated this work.

The second author was partially supported by CNPq, FAPESP and FINEP

The third author was supported partially supported by $\mathrm{CNPq}$.

(C)2000 American Mathematical Society 
In a recent paper [9, the author explored the validity of the Similarity Principle for the following three nonelliptic vector fields:

$$
L_{1}=\frac{\partial}{\partial t}-3 i t^{2} \frac{\partial}{\partial x}, \quad L_{2}=\frac{\partial}{\partial t}-i x \frac{\partial}{\partial x}, \quad \text { and } \quad M=\frac{\partial}{\partial t}-i t \frac{\partial}{\partial x} .
$$

It is proved in [9] that the Similarity Principle is valid for $L_{1}$ and $L_{2}$ in the following sense: if $w$ is a solution of $L_{j} w=A w+B \bar{w} \quad(j=1,2)$, then $w$ has the form

$$
w=e^{g} h,
$$

where $L_{j} h=0$. It is also shown that the Similarity Principle fails for the Mizohata vector field $M$. The vector fields $\frac{\partial}{\partial \bar{z}}, L_{1}$ and $L_{2}$ are locally solvable while $\mathrm{M}$ is not.

In this paper we will prove a generalized similarity principle for solutions of (1.1) where $L$ belongs to a class of locally solvable vector fields (Theorem 3.1). This result is applied to establish uniqueness in the Cauchy problem for semilinear equations

$$
L w=f(t, x, w)
$$

where $f$ is assumed to have bounded first derivatives and $w$ is in $L_{\text {loc }}^{p}, p>1$ (Theorem 4.1) . When the vector field $L$ is also hypoelliptic and subelliptic, we get a similarity principle closer in character to the classical one (see subsection 4.2).

\section{A Class of A PRIORI ESTIMATES}

We recall some facts about the real Hardy space $H^{1}(\mathbb{R}) \subset L^{1}(\mathbb{R})$, a particular instance of the spaces introduced by Stein and Weiss in [14], and its semi-local version $h^{1}(\mathbb{R})$ introduced by Goldberg [6]. In many situations $H^{1}(\mathbb{R})$ is an advantageous substitute for $L^{1}(\mathbb{R})$ [13], as the latter does not behave well in many respects, for instance, concerning the continuity of singular integral operators. Let's choose a function $\Phi \geq 0 \in C_{c}^{\infty}([-1 / 2,1 / 2])$, with $\int \Phi d z=1$. Write $\Phi_{\varepsilon}(z)=\varepsilon^{-1} \Phi(z / \varepsilon)$, $z \in \mathbb{R}$, and set

$$
M_{\Phi} f(z)=\sup _{0<\varepsilon<\infty}\left|\left(\Phi_{\varepsilon} * f\right)(z)\right|
$$

Then [13]

$$
H^{1}(\mathbb{R})=\left\{f \in L^{1}(\mathbb{R}): \quad M_{\Phi} f \in L^{1}(\mathbb{R})\right\} .
$$

A space of distributions is called semi-local if it is invariant under multiplication by test functions. The space $H^{1}(\mathbb{R})$ is not: $\psi u$ may not belong to $H^{1}(\mathbb{R})$ for $\psi \in C_{c}^{\infty}(\mathbb{R})$ and $u \in H^{1}(\mathbb{R})$. A way around this is the definition of the semi-local (or localizable) Hardy space - better suited for the study of PDE's- $h^{1}(\mathbb{R})$ ([6] [13]) by means of the truncated maximal function

$$
\begin{gathered}
m_{\Phi} f(z)=\sup _{0<\varepsilon \leq 1}\left|\left(\Phi_{\varepsilon} * f\right)(z)\right| ; \\
h^{1}(\mathbb{R})=\left\{f \in \mathcal{S}^{\prime}(\mathbb{R}): \quad m_{\Phi} f \in L^{1}(\mathbb{R})\right\},
\end{gathered}
$$

which is stable under multiplication by test functions. Moreover, $h^{1}(\mathbb{R})$ is a Banach space with the norm

$$
\|f\|_{h^{1}}=\left\|m_{\Phi} f\right\|_{L^{1}}
$$

and $H^{1} \subset h^{1} \subset L^{1}$. We now describe the atomic decomposition of $h^{1}$ ([6], [13]). An $h^{1}(\mathbb{R})$ atom is a bounded, compactly supported function $\alpha(z)$ satisfying the following properties: there exist an interval $Q$ containing the support of $\alpha$ such that 
(1) $|\alpha(z)| \leq|Q|^{-1}$, a.e., with $|Q|$ denoting the Lebesgue measure of $Q$;

(2) if the length of $Q$ is $<1$, we further require that $\int \alpha(z) d z=0$.

Any $f \in h^{1}$ can be written as an infinite linear combination of $h^{1}$ atoms, more precisely, there exist scalars $\lambda_{j}$ and $h^{1}$ atoms $\alpha_{j}$ such that the series $\sum_{j} \lambda_{j} \alpha_{j}$ converges in $h^{1}$ to $f$. Furthermore, $\|f\|_{h^{1}} \sim \inf \sum_{j}\left|\lambda_{j}\right|$, where the infimum is taken over all atomic representations. Another useful fact is that the atoms may be assumed to be smooth functions. A simple consequence of the atomic decomposition is that $h^{1}(\mathbb{R})$ is stable under multiplication by Lipschitz functions $a(x)$ : if $\alpha$ satisfies (1) with $|Q| \geq 1$, it follows that $a(x) \alpha(x) /\|a\|_{L_{\infty}}$ also does. If $|Q|<1$ and the center of $Q$ is $x_{0}$, we may write $a(x) \alpha(x)=a\left(x_{0}\right) \alpha(x)+\left(a(x)-a\left(x_{0}\right)\right) \alpha(x)=\beta_{1}(x)+\beta_{2}(x)$. Then $\beta_{1}(x) /\|a\|_{L^{\infty}}$ satisfies (1) and (2) (with the same $Q$ ) while $\beta_{2}(x) / K$ satisfies (1) for the interval $Q^{\prime}$ of center $x_{0}$ and length 1 , where $K$ is the Lipschitz constant of $a(x)$. It follows that $f \mapsto a f$ has norm $\leq\|a\|_{L^{\infty}}+K$ in $h_{1}(\mathbb{R})$.

Consider now a first-order linear differential operator in two variables

$$
L=\frac{\partial}{\partial t}+i b(t, x) \frac{\partial}{\partial x}+c(t, x), \quad x \in \mathbb{R}, \quad|t|<T .
$$

We write $\Omega_{T}=[-T, T] \times \mathbb{R}$ and assume that

i) $c(t, x)$ is smooth, $b(t, x)$ is smooth, real and nonnegative,

ii) all derivatives of $b(t, x)$ and $c(t, x)$ are uniformly bounded.

The fact that $b(t, x) \geq 0$ implies that $L$ has a left parametrix $K$ whose form we briefly describe. Its construction is analogous to that of a right parametrix, a detailed account of which can be found in [7] and [8]. In fact, the transpose of a right parametrix for the transpose of $L$ gives a left parametrix for $L$. The basic property of $K$ is that (for a small $T>0$ )

$$
K L u=u+R u, \quad u \in C_{c}^{\infty}\left(\Omega_{T}\right),
$$

where $R$ is a regularizing operator. The operator $K$ may be written as $K=$ $K^{+}+K^{-}$, with

$$
K^{+} f(t, x)=\int_{-T}^{t} A_{t, t^{\prime}}^{+}(f \circ \psi) d t^{\prime}
$$

and

$$
K^{-} f(t, x)=\int_{T}^{t} A_{t, t^{\prime}}^{-}(f \circ \psi) d t^{\prime}
$$

Here, $A_{t, t^{\prime}}^{ \pm}$are pseudo-differential operators in $\mathbb{R}_{x}$ for fixed $t, t^{\prime}$ of order zero and type $(\rho, \delta)=(1,1 / 2)$. Furthermore, they depend smoothly on $t$ and $t^{\prime}$. For all $t$ and $t^{\prime}$ the function $x \mapsto \psi=\psi\left(t, x, t^{\prime}\right)$ is a diffeomorphism of $\mathbb{R}_{x}$ onto $\mathbb{R}_{x}$ with bounded derivatives. It is known (see e.g. 1], Thm. 3.2) that

$$
\left\|A_{t, t^{\prime}}^{ \pm} f(x)\right\|_{L^{1}(\mathbb{R})} \leq C\left(t, t^{\prime}\right)\|f\|_{H^{1}(\mathbb{R})}, \quad f \in H^{1}(\mathbb{R}),
$$

where the constants $C\left(t, t^{\prime}\right)$ depend on a finite number of seminorms of the symbol space $S_{1,1 / 2}^{0}(\mathbb{R})$. The argument can be adapted to obtain

$$
\left\|A_{t, t^{\prime}}^{ \pm} f(x)\right\|_{L^{1}(\mathbb{R})} \leq C\left(t, t^{\prime}\right)\|f\|_{h^{1}(\mathbb{R})}, \quad f \in h^{1}(\mathbb{R}) .
$$

Indeed, if $A$ is any pseudo-differential operator of order zero and type $(\rho, \delta)=$ $(1,1 / 2)$ and $\alpha$ is a "small $h^{1}$-atom", i.e., it has vanishing mean and is supported in a minimal interval $Q$ of length $|Q| \leq 1$, it belongs to $H^{1}$ and the quantities $|Q|\|\alpha\|_{L^{\infty}},\|\alpha\|_{H^{1}}$ and $\|\alpha\|_{h^{1}}$ are all equivalent and bounded by a fixed constant. 
Hence, $\|A \alpha\|_{L^{1}} \leq C$, by the $H^{1}$ estimate. Assume now that $|Q|>1$ with center $x_{0}$ and denote by $Q^{*}$ the interval that has the same center as $Q$ but double length. As usual

$$
\int_{Q^{*}}|A \alpha| d x \leq\left|Q^{*}\right|^{1 / 2}\|A \alpha\|_{L^{2}} \leq C|Q|^{1 / 2}\|\alpha\|_{L^{2}} \leq C^{\prime}
$$

because $A$ is bounded in $L^{2}$. On the other hand, if $k(x, y)$ is the kernel of $A$, $|x-y|^{2}|k(x, y)| \leq C$ whenever $x \neq y$. Hence

$$
\begin{aligned}
\int_{\mathbb{R} \backslash Q^{*}}|A \alpha| d x & =\int_{\mathbb{R} \backslash Q^{*}}\left|\int k(x, y) \alpha(y) d y\right| d x \\
& \leq C\|\alpha\|_{L^{\infty}}|Q| \int_{\left|x-x_{0}\right|>1}\left|x-x_{0}\right|^{-2} d x \leq C^{\prime} .
\end{aligned}
$$

Thus $\|A \alpha\|_{L^{1}} \leq C$ for any $h^{1}$-atom, which implies that $\|A f\|_{L^{1}} \leq C\|f\|_{h^{1}}$ for any $f \in h^{1}$.

Since the symbols $a^{ \pm}\left(x, \xi ; t, t^{\prime}\right)$ of $A_{t, t^{\prime}}^{ \pm}$depend smoothly on the parameters $\left(t, t^{\prime}\right)$ the set $\left\{C\left(t, t^{\prime}\right) ; t, t^{\prime} \in[-T, T]\right\}$ is bounded and we get

$$
\left\|A_{t, t^{\prime}}^{ \pm} f(x)\right\|_{L^{1}(\mathbb{R})} \leq C\|f\|_{h^{1}(\mathbb{R})}, \quad f \in h^{1}(\mathbb{R}),
$$

with a uniform $C$.

Integrating in $t$ we easily derive

$$
\|K u\|_{L^{1}\left(\Omega_{T}\right)} \leq C T\|u(t, \psi(x))\|_{L^{1}\left([T, T], h^{1}\left(\mathbb{R}_{x}\right)\right)},
$$

for any $u \in C_{c}^{\infty}\left(\Omega_{T}\right)$. If $f(x) \in C_{c}^{\infty}([-T, T])$, the diffeomorphism invariance of $h^{1}$ ([6]) implies that $\|f \circ \psi\|_{h^{1}(\mathbb{R})} \leq C\|f\|_{h^{1}(\mathbb{R})}$. Thus

$$
\|K u\|_{L^{1}\left(Q_{T}\right)} \leq C T\|u\|_{L^{1}\left([T, T], h^{1}\left(\mathbb{R}_{x}\right)\right)},
$$

for any $u \in C_{c}^{\infty}\left(Q_{T}\right)$, where $Q_{T}=[-T, T] \times[-T, T]$.

The expression for $R$ is similar, with operators $B_{t, t^{\prime}}^{ \pm} \in S^{-\infty}(\mathbb{R})$ which, in particular, take $L^{1}(\mathbb{R})$ into itself and it is easy to obtain

$$
\|R u\|_{L^{1}\left(Q_{T}\right)} \leq C T\|u\|_{L^{1}\left(Q_{T}\right)} .
$$

It follows from $(2.2),(2.3)$ and (2.4) that

$$
\left.\|u\|_{L^{1}\left(Q_{T}\right)} \leq C T\left(\|L u\|_{L^{1}\left([-T, T], h^{1}\left(\mathbb{R}_{x}\right)\right.}\right)+\|u\|_{L^{1}\left(Q_{T}\right)}\right) .
$$

Taking $T>0$ small enough so that $C T<1 / 2$ we get

$$
\|u\|_{L^{1}\left(Q_{T}\right)} \leq C T\|L u\|_{L^{1}\left([-T, T], h^{1}\left(\mathbb{R}_{x}\right)\right)}, \quad u \in C_{c}^{\infty}\left(Q_{T}\right) .
$$

We have proved

Theorem 2.1. Let the operator $L$ with smooth coefficients given by (2.1) satisfy i) and ii). Then there exist constants $C>0$ and $T_{0}$ such that

$$
\|u\|_{L^{1}\left(Q_{T}\right)} \leq C T\|L u\|_{L^{1}\left([-T, T], h^{1}\left(\mathbb{R}_{x}\right)\right)},
$$

for all $u \in C_{c}^{\infty}\left(Q_{T}\right), 0<T \leq T_{0}$.

The a priori inequality (2.5) has a standard duality consequence which we now describe. The dual of $h^{1}(\mathbb{R})$, denoted by $\operatorname{bmo}(\mathbb{R})$, may be identified $([6])$ with the space of locally integrable functions $f(x)$ such that $\sup _{|Q|<1}|Q|^{-1} \int_{Q}\left|f-f_{Q}\right|<\infty$ and $\sup _{|Q| \geq 1}|Q|^{-1} \int_{Q}|f|<\infty$, where we have denoted by $Q$ an arbitrary interval and by $f_{Q}$ the mean of $f$ on $Q$. In particular bmo $(\mathbb{R})$ is contained in $\operatorname{BMO}(\mathbb{R})$, 
the space of bounded mean oscillation functions (to avoid the difficulty that the usual BMO space is made up of classes modulo constant functions rather than true functions, i.e., the usual BMO "norm" of a constant is zero, it is convenient to add to the usual "norm" the term $\int_{0}^{1}|f(x)| d x$, so BMO becomes a true space of functions). Then, (2.5) implies local solvability in $L^{\infty}\left([-T, T], \operatorname{bmo}\left(\mathbb{R}_{x}\right)\right)$ for the formal transpose $L^{t}$. Now, $L$ and $-L^{t}$ have the same principal part, so $L$ and $-L^{t}$ satisfy simultaneously the hypotheses of Theorem 2.1. Summing up,

Corollary 2.2. Let the operator

$$
L=\frac{\partial}{\partial t}+i b(t, x) \frac{\partial}{\partial x}+c(t, x)
$$

satisfy i) and ii). There is a neighborhood $U$ of the origin such that for every function $f \in L^{\infty}\left(Q_{T}\right)$ there exists a function $u \in L^{\infty}\left([-T, T]\right.$, bmo $\left.\left(\mathbb{R}_{x}\right)\right)$ which solves $L u=f$ in $U$, with norm

$$
\|u\|_{L^{\infty}\left([-T, T], \mathrm{bmo}\left(\mathbb{R}_{x}\right)\right)} \leq C T\|f\|_{L^{\infty}\left(Q_{T}\right)} .
$$

In particular, if $f \in L^{\infty}\left(\mathbb{R}^{2}\right)$ we may solve the equation $L u=f$ in a disc $U$, the solution $u \in L^{\infty}\left([-T, T], \mathrm{BMO}\left(\mathbb{R}_{x}\right)\right)$ satisfying

$$
\|u\|_{L^{\infty}\left([-T, T], \operatorname{BMO}\left(\mathbb{R}_{x}\right)\right)} \leq C\|f\|_{L^{\infty}}
$$

and $C \rightarrow 0$ as the diameter of $U$ shrinks to zero.

\section{Generalized Similarity PRINCIPLE}

Suppose that $L=\frac{\partial}{\partial t}+i b(t, x) \frac{\partial}{\partial x}$ is a smooth vector field defined in some open subset $\Omega$ of the plane, with $b(t, x)$ having a consistent sign. Assume $A$ and $B$ are $L^{\infty}$ functions, $\omega \in L_{\mathrm{loc}}^{p}(\Omega)$ for some $1<p<\infty$, and that

$$
L \omega=A \omega+B \bar{\omega}
$$

in the sense of distributions. The next theorem describes an expression for $\omega$ that involves the space $X=L^{\infty}\left(\mathbb{R}_{t} ; \operatorname{bmo}\left(\mathbb{R}_{x}\right)\right)$ of measurable functions $u(t, x)$ such that for almost every $t \in \mathbb{R} x \mapsto u(t, x) \in \operatorname{bmo}(\mathbb{R})$ and $\|u(t, \cdot)\|_{\text {bmo }} \leq C<\infty$ for a.e. $t \in \mathbb{R}$. Observe that $X$ is invariant under multiplication by test functions and $u \in X \Rightarrow|u| \in X$, because $\mathrm{bmo}(\mathbb{R})$ already has these properties.

Theorem 3.1. Let $L, p, A, B$ and $\omega$ satisfying (3.1) be as above. Every point of $\Omega$ has a neighborhood $\Omega^{\prime}$ where $\omega$ may be written as

$$
\omega=e^{g} h,
$$

with $h \in L_{\text {loc }}^{p^{\prime}}\left(\Omega^{\prime}\right)$ satisfying $L h=0$ in $\Omega^{\prime}, g \in X$ and $e^{g} \in L_{\text {loc }}^{q^{\prime}}\left(\Omega^{\prime}\right)$ for some $p^{\prime} \in[1, p]$ and $q^{\prime} \geq p^{\prime} /\left(p^{\prime}-1\right)$. In addition, $p^{\prime}$ may be chosen arbitrarily close to $p$.

We will need the following lemma in the proof of Theorem 3.1.

\section{Lemma 3.2.}

i) Let $p, q \in(1, \infty), 1 / p+1 / q=1, u, f \in L_{\mathrm{loc}}^{p}(\Omega), v, g \in L_{\mathrm{loc}}^{q}(\Omega)$, and assume that $L u=f$ and $L v=g$. Then,

$$
L(u v)=f v+u g .
$$

ii) Let $p \in(1, \infty]$ and assume that $g \in X$ satisfies $L g \in L^{p}(\Omega)$. If $\|g\|_{X}$ is sufficiently small,

$$
L\left(e^{g}\right)=e^{g} L g, \quad \text { in } \Omega .
$$


Proof. i) Using a cut-off function we may assume that $u$ and $v$ are compactly supported in $\Omega$ and use regularizations $u_{\varepsilon}=\phi_{\varepsilon} * u, v_{\varepsilon}=\phi_{\varepsilon} * v$ and set $f_{\varepsilon}=L u_{\varepsilon}$ and $g_{\varepsilon}=L v_{\varepsilon}$. Of course, $u_{\varepsilon} \rightarrow u$ in $L^{p}$ and $v_{\varepsilon} \rightarrow v$ in $L^{q}$ while Friedrichs' lemma implies that $f_{\varepsilon} \rightarrow f$ in $L^{p}$ and $g_{\varepsilon} \rightarrow g$ in $L^{q}$. Then, writing $u g-u_{\varepsilon} g_{\varepsilon}=$ $u\left(g-g_{\varepsilon}\right)+\left(u-u_{\varepsilon}\right) g_{\varepsilon}$ and applying Hölder's inequality to each term we check that $u_{\varepsilon} g_{\varepsilon} \rightarrow u g$ in $L^{1}$. Similarly, $f_{\varepsilon} v_{\varepsilon} \rightarrow f v$ and $u_{\varepsilon} v_{\varepsilon} \rightarrow u v$ in $L^{1}$. Now the claim follows, since $L\left(u_{\varepsilon} v_{\varepsilon}\right)=u_{\varepsilon} g_{\varepsilon}+v_{\varepsilon} f_{\varepsilon}$.

ii) It is enough to prove (3.3) in a neighborhood of an arbitrary point of $\Omega$. If $\chi$ is a test function and we set $g_{1}=\chi g$, it follows that $g_{1} \in X$ and $L g_{1}=\chi L g+g L \chi \in$ $L^{p}$, since $X \subset L_{\mathrm{loc}}^{p}\left(\mathbb{R}^{2}\right)$. Hence, we may assume from the start that $g$ is compactly supported. In particular, $g \in L^{\infty} \Rightarrow g \in L^{q}, 1 \leq q \leq \infty$ and it is enough to prove (3.3) assuming that $p<\infty$. Let $\phi(x) \geq 0, x \in \mathbb{R}$, be a test function with $\int \phi=1$ and set $J_{\varepsilon}^{x} u=\phi_{\varepsilon} * u, u \in \mathcal{S}^{\prime}(\mathbb{R}), 0<\varepsilon<1$. If $u \in h^{1}(\mathbb{R})$,

$$
\begin{aligned}
\left\|J_{\varepsilon}^{x} u\right\|_{h^{1}} & =\left\|m_{\Phi} J_{\varepsilon}^{x} u\right\|_{L^{1}}=\left\|\sup _{0<t<1}\left|\Phi_{t} *\left(\phi_{\varepsilon} * u\right)\right|\right\|_{L^{1}} \\
& \leq\left\|\sup _{0<t<1} \phi_{\varepsilon} *\left|\Phi_{t} * u\right|\right\|_{L^{1}} \leq\left\|\phi_{\varepsilon} * m_{\Phi} u\right\|_{L^{1}}=\left\|m_{\Phi} u\right\|_{L^{1}}=\|u\|_{h^{1}} .
\end{aligned}
$$

The duality between $h^{1}$ and bmo then shows that

$$
\left\|J_{\varepsilon}^{x} u\right\|_{\mathrm{bmo}} \leq\|u\|_{\mathrm{bmo}}, \quad u \in \mathrm{bmo}(\mathbb{R}) .
$$

Now set $\psi(t, x)=\phi(t) \phi(x)$ and $g_{\varepsilon}=\psi_{\varepsilon} * g=J_{\varepsilon}^{t} J_{\varepsilon}^{x} g$. Since $g$ has compact support, $g \in L^{p^{\prime}}\left(\mathbb{R}^{2}\right)$ for any $p^{\prime}<\infty$ and $g_{\varepsilon} \rightarrow g$ in $L^{p^{\prime}}\left(\mathbb{R}^{2}\right)$. By Friedrichs' lemma, $L\left(g_{\varepsilon}\right) \rightarrow$ $L g$ in $L^{p}\left(\mathbb{R}^{2}\right)$. Let $Q$ be a cube that contains the support of $g$. Using a standard consequence of the John-Nirenberg inequality in the variable $x$ and integrating the result with respect to $t$, we see that given $q<\infty,\left\|e^{|g|}\right\|_{L^{q}(Q)} \leq C\left(q,\|g\|_{X}\right)$ and $\left\|e^{\left|g_{\varepsilon}\right|}\right\|_{L^{q}(Q)} \leq C\left(q,\left\|g_{\varepsilon}\right\|_{X}\right) \leq C\left(q,\|g\|_{X}\right)$, provided $\|g\|_{X}$ is sufficiently small. We have used the fact that $g \in X \Rightarrow|g| \in X$ and $\||g|\|_{X} \leq C\|g\|_{X}$.

Using Hölder's inequality on the right hand side of the estimate

$$
\left|e^{g(t, x)}-e^{g_{\varepsilon}(t, x)}\right| \leq e^{|g(t, x)|} e^{\left|g_{\varepsilon}(t, x)\right|}\left|g(t, x)-g_{\varepsilon}(t, x)\right|
$$

we get, assuming that $\|g\|_{X}$ is sufficiently small,

$$
\left\|e^{g}-e^{g_{\varepsilon}}\right\|_{L^{q}(Q)} \leq\left\|e^{|g|}\right\|_{L^{3 q}(Q)}\left\|e^{\left|g_{\varepsilon}\right|}\right\|_{L^{3 q}(Q)}\left\|g-g_{\varepsilon}\right\|_{L^{3 q}(Q)} \leq C\left\|g-g_{\varepsilon}\right\|_{L^{3 q}(Q)} .
$$

We conclude that $L\left(e^{g_{\varepsilon}}\right)=e^{g_{\varepsilon}} L\left(g_{\varepsilon}\right)$ converges to $e^{g} L(g)$ in $L_{\text {loc }}^{1}(\Omega)$ if we choose $q=p /(p-1)$. This proves $(3.3)$.

Now, we return to the proof of Theorem 3.1 .

Proof. Consider a neighborhood $\Omega^{\prime}$ of the given point of $\Omega$ where we may solve the equation

$$
L g=A+B \frac{\chi \bar{\omega}}{\omega} \text { in } \Omega^{\prime}
$$

with $\chi$ representing the characteristic function of the set $\{\omega(x) \neq 0\}$. The right hand side is bounded. Therefore, Corollary 2.2 implies that shrinking $\Omega^{\prime}$ we may solve (3.4) with $\|g\|_{X}$ as small as we wish. Then, if we set $h=e^{-g} \omega$ and use the Leibniz and chain rules (3.2) and (3.3) provided by Lemma 3.2 we get

$$
L h=e^{-g}(L \omega-\omega L g)=e^{-g}\left(A \omega+B \bar{\omega}-\omega\left(A+B \frac{\chi \bar{\omega}}{\omega}\right)=0 .\right.
$$


Thus, $\omega=e^{g} h$ as we wished to prove. Observe that by shrinking $\Omega^{\prime}$ we may take $e^{-g} \in L^{q^{\prime}}$ with $q^{\prime}$ arbitrarily large and this implies that $h \in L^{p^{\prime}}\left(\Omega^{\prime}\right)$ with $p^{\prime}<p$ arbitrarily close to $p$. This proves Theorem 3.1.

\section{Applications}

4.1. Uniqueness in the Cauchy problem. We consider now a locally solvable vector field $L$. After a choice of appropiate coordinates and multiplication by a nonvanishing factor, near a central point $0, L$ has the form

$$
L=\frac{\partial}{\partial t}-i b(t, x) \frac{\partial}{\partial x}, \quad \text { with } b(t, x) \text { real-valued. }
$$

The local solvability of $L$ is then equivalent to the statement ([10]): for each $x$, the function $t \longmapsto b(t, x)$ does not change sign.

Theorem 4.1. Suppose $L$ is locally solvable and $f(t, x, \zeta, \bar{\zeta})$ is a complex-valued function with bounded derivatives for $t, x$ real and $\zeta$ complex. Let $\Sigma$ be a noncharacteristic curve for $L$ in an open set $\Omega$. If $u, w \in L_{\mathrm{loc}}^{p},(p>1)$, solve

$$
L u=f(t, x, u, \bar{u}), \quad L w=f(t, x, w, \bar{w}) \quad \text { in } \Omega, \quad \text { and } \quad u_{\left.\right|_{\Sigma}}=w_{\left.\right|_{\Sigma}} .
$$

Then $u \equiv w$ in a neighborhood of $\Sigma$.

Proof. The proof of the theorem follows from the following lemma, after linearizing $f(t, x, w, \bar{w})-f(t, x, u, \bar{u})$.

Lemma 4.2. Suppose $L$ is locally solvable, $A$ and $B$ are in $L^{\infty}, w \in L_{\text {loc }}^{p}(p>1)$,

$$
L w=A w+B \bar{w} \quad \text { in } \Omega, \text { and } w_{\left.\right|_{\Sigma}}=0 \text {, }
$$

where $\Sigma \subset \Omega$ is a noncharacteristic curve for $L$. Then $w \equiv 0$ in a neighborhood of $\Sigma$.

Proof. We may assume that $\Omega=\{(t, x) ;|t|<1,|x|<1\}, \Sigma=\{t=0\}$, and $L=$ $\frac{\partial}{\partial t}-i b(t, x) \frac{\partial}{\partial x}$. Let $N=\{x \in(-1,1) ; b(t, x)=0,|t|<1\}$. Pick $y_{0} \in(-1,1) \backslash N$. There exists $\varepsilon>0$ such that either $b \geq 0$ or $b \leq 0$ on $Q=\left\{(t, y) ;|t|<1,\left|y-y_{0}\right|<\right.$ $\varepsilon\}$. Define $V(t, x)$ in $Q$ by

$$
V(t, x)=\left\{\begin{array}{cc}
w(t, x), & t>0 \\
0, & t<0
\end{array}\right.
$$

We will show that $L V=A V+B \bar{V}$ in $Q$. Let $\psi \in C_{c}^{\infty}(Q)$. Let $\left\{\varphi_{k}(t)\right\}$ be a sequence of $C^{\infty}$ functions on $\mathbb{R}$ such that $0 \leq \varphi_{k} \leq 1$,

$$
\varphi_{k}(t)= \begin{cases}1, & t>\frac{-1}{2 k}, \\ 0, & t<\frac{-1}{k},\end{cases}
$$

and $\left\|\varphi_{k}^{\prime}\right\|_{L^{\infty}} \leq C k$ for some $C$ independent of $k$. For any $k$ we have

$$
\begin{aligned}
\langle L V, \psi(t, x)\rangle & =\left\langle L V, \varphi_{k}(t) \psi(t, x)\right\rangle \\
& =\iint V^{t} L\left(\varphi_{k} \psi\right) d t d x \\
& =\iint w^{t} L\left(\varphi_{k} \psi\right) d t d x-\int_{-1}^{1} \int_{-\frac{1}{k}}^{0} w^{t} L\left(\varphi_{k} \psi\right) d t d x \\
& =\iint(A w+B \bar{w}) \varphi_{k} \psi d t d x-\int_{-1}^{1} \int_{-\frac{1}{k}}^{0} w^{t} L\left(\varphi_{k} \psi\right) d t d x
\end{aligned}
$$


Since $k \rightarrow \infty$, the term

$$
\iint(A w+B \bar{w}) \varphi_{k} \psi d t d x \quad \text { converges to } \quad\langle A V+B \bar{V}, \psi\rangle .
$$

Since $\left|\varphi_{k}^{\prime}(t)\right| \leq C k$ for all $k$ and the trace of $w$ on $\{t=0\}$ is 0 , the integral

$$
\int_{-1}^{1} \int_{-\frac{1}{k}}^{0} w^{t} L\left(\varphi_{k} \psi\right) d t d x \text { goes to } 0, \text { as } k \rightarrow \infty \text {. }
$$

Thus $L V=A V+B \bar{V}$ in $Q$.

Observe that in $Q, L$ is of the kind to which we can apply the results of section 3. Therefore, there is a neighborhood $U_{1}$ of $\left(0, y_{0}\right)$ in which

$$
V=e^{g} h \quad \text { with } \quad L h=0 .
$$

But then $h=0$ on $\left\{(t, x) \in U_{1} ; t<0\right\}$. By the theorem on propagation of zeroes for homogeneous solutions (see [15]) it follows that $h=0$ in $U_{1}$, and hence $V=0$ in $U_{1}$.

Next, if $0<t_{0}<1$, by covering the segment from $\left(0, y_{0}\right)$ to $\left(t_{0}, y_{0}\right)$ by a chain of open sets in each of which the factorization of Theorem 3.1 is valid, and applying the theorem on the propagation of zeroes for homogeneous solutions, we conclude:

$$
V \equiv 0 \quad \text { in a neighborhood of }(-1,1) \times\left\{y_{0}\right\}
$$

Hence $w \equiv 0$ in a neighborhood of $(0,1) \times\left\{y_{0}\right\}$. By repeating this reasoning, we conclude that $w \equiv 0$ in a neighborhood of $(-1,1) \times\left\{y_{1}\right\}$, for any $y_{1}$ such that $\left|y_{1}-z_{0}\right|<\varepsilon$. Hence $w \equiv 0$ on $Q$.

Suppose now that $\varphi \in C_{c}^{\infty}(\Omega)$. Then since $\left\{x \in N ; \frac{\partial b}{\partial x}(t, x) \neq 0\right.$, for some $\left.t\right\}$ is discrete, we get

$$
\begin{aligned}
\langle L w, \varphi(t, x)\rangle & =\int_{N} \int_{-1}^{1} w(t, x)^{t} L \varphi(t, x) d t d x \\
& =-\int_{N} \int_{-1}^{1} w(t, x) \frac{\partial \varphi}{\partial t}(t, x) d t d x \\
& =\left\langle\frac{\partial w}{\partial t}, \varphi(t, x)\right\rangle .
\end{aligned}
$$

Therefore, $\frac{\partial w}{\partial t}=A w+B \bar{w}$ in $\Omega$. But $\frac{\partial}{\partial t}$ is of the type studied in section 3 and $w(0, x)=0$. Hence by the same arguments used for $V$ we conclude:

$$
w \equiv 0 \quad \text { in a neigborhood of } \Sigma=\{t=0\},
$$

completing the proof of Lemma 4.2.

Using the proof of Theorem 4.1, we will next prove the following corollary which extends the theorem to higher dimensions.

Corollary 4.3. Suppose $L$ is locally solvable in a neighborhood $\Omega$ of the origin in $\mathbb{R}^{n}, f(x, \zeta, \bar{\zeta})$ is a complex valued function with bounded derivatives for $x$ in $\mathbb{R}^{n}$ and $\zeta$ complex. Let $\Sigma$ be a noncharacteristic hypersurface in $\Omega$ passing through the origin. If $u, w \in L_{\mathrm{loc}}^{p}(p>1)$ solve

$$
L u=f(x, u, \bar{u}), \quad L w=f(x, w, \bar{w}) \quad \text { in } U, \quad \text { and } \quad u_{\left.\right|_{\Sigma}}=w_{\left.\right|_{\Sigma}},
$$

then $u \equiv w$ in a neighborhood of $\Sigma$. 
Proof. The local solvability of $L$ implies that we can find coordinates $(t, x)$ in some neighborhood $U=(-\delta, \delta) \times U_{1}$ of the origin in $\mathbb{R}^{n}$ in which

$$
L=\frac{\partial}{\partial t}+i \sum_{k=1}^{n-1} b_{k}(t, x) \frac{\partial}{\partial x_{k}}
$$

where each $b_{k}$ is real-valued, smooth on $U$ and that for each $x \in U_{1}$, the function

$$
t \longmapsto\left(b_{1}(t, x), \ldots, b_{n-1}(t, x)\right)=b(t, x)
$$

never changes direction. We may also assume that in $U$ the surface $\Sigma$ is given by $t=0$. As before if we let $v=u-w$, then $L v=A v+B \bar{v}$ and the trace of $v$ on $\Sigma$ is zero. Let $N=\left\{x \in U_{1}: b(t, x)=0,|t|<\delta\right\}$. Fix $x_{0} \notin N$. We can get a unit vector field $V(x)$ in $x$ space, smooth outside $N$ such that

$$
L=\frac{\partial}{\partial t}+i|b(t, x)| V(x) \mid
$$

Since $V$ is smooth and nonzero near $x_{0}$, after a diffeomorphism in $x$ space near $x_{0}$, we get coordinates $(t, y)$ in which $L$ takes the form

$$
L=\frac{\partial}{\partial t}+i c\left(t, y_{1}, y^{\prime}\right) \frac{\partial}{\partial y_{1}}
$$

near some point $\left(0, y_{0}\right)$. Moreover, the function $c\left(t, y_{1}, y^{\prime}\right) \geq 0$. If we continue to use the same notation for $v, A$ and $B$ in these coordinates, we have $L v=A v+B \bar{v}$ and $v(0, y)=0$. Now for each $y^{\prime}$ fixed, $L$ is a planar vector field to which the similarity principle applies. Note also that since $v \in L_{\text {loc }}^{p}$, there is a set $S$ of measure zero in $y^{\prime}$ space such that for any $y^{\prime}$ outside $S$, the function $\left(t, y_{1}\right) \longmapsto v\left(t, y^{\prime}, y_{1}\right)$ is a solution of the equation with the planar vector field $L$ frozen at $y^{\prime}$. We can therefore proceed as in the proof of Lemma 4.2 to conclude the proof of this corollary.

We note that the two-dimensional nature of a locally solvable vector field was used in other works (see for example, [15]).

4.2. The hypoelliptic case. In the following applications, we will assume that the vector field $L$ is hypoelliptic and consider the equation $L w=A w+B \bar{w}$, where $A$ and $B$ are smooth. It is well known (see [15]) that such a vector field is hypocomplex, equivalently, it has local first integrals that are homeomorphisms. It follows that a hypoelliptic vector field is of the kind considered in Section 2. An earlier proof of this latter fact appeared in the Appendix in [7].

Corollary 4.4. Suppose $w$ is not identically zero. Assume that $h=e^{g} w$ in some open set $\Omega$ where $L h=0$ and $e^{|g|} \in L^{2}(\Omega)$. Then

a) $w$ and $h$ have the same zeroes in $\Omega$.

b) at a zero, $w$ and $h$ vanish to the same order. Thus since $L$ is hypocomplex, the zeroes of $w$ are isolated and of finite order.

Proof. To prove (a) suppose that $w(p)=0$ and $h(p) \neq 0$. Then for $z$ near $p$,

$$
0<\frac{|h(p)|}{2} \leq c\left|e^{g(z)}\right||z-p| \text { for some } c>0 .
$$

But then near $p$,

$$
\left|e^{2 g(z)}\right| \geq \frac{c_{1}}{|z-p|^{2}} \quad \text { for some } c_{1}>0
$$


contradicting the fact that $e^{g} \in L^{2}(\Omega)$. Hence if $w(p)=0$ and $p \in \Omega$, then $h(p)=0$. Conversely, if $h(p)=0$, since $e^{-g} \in L^{2}(\Omega)$, we must have $w(p)=0$.

To prove (b) we first prove the following claim. (We will also use this fact in Corollary 4.6.)

From now on we will replace the variable " $t$ " by " $y$ ".

Assume that $L=\frac{\partial}{\partial y}+i b(x, y) \frac{\partial}{\partial x}, \quad b(0,0)=0$. (Note that if $\mathrm{b}(0,0)$ is not zero, we would have an elliptic vector field for which the corollary is valid.)

Claim 4.5. Suppose $n+l$ is the smallest such that $D_{x}^{n} D_{y}^{l} w(0) \neq 0$. Then $l=0$.

To prove the claim, suppose in fact $l \geq 1$. Then applying the operator $D_{x}^{n} D_{y}^{l-1}$ to the equation

$$
D_{y} w+i b(x, y) D_{x} w=A w+B \bar{w}
$$

yields

$$
\begin{gathered}
D_{x}^{n} D_{y}^{l} w+i \sum_{s, t} c_{s t}\left(D_{x}^{n-t} D_{y}^{l-1-s} b\right)\left(D_{x}^{t+1} D_{y}^{s} w\right) \\
=\sum_{s, t} c_{s t}\left(D_{x}^{n-t} D_{y}^{l-1-s} A\right)\left(D_{x}^{t} D_{y}^{s} w\right) \\
\quad+\sum_{s, t} c_{s t}\left(D_{x}^{n-t} D_{y}^{l-1-s} B\right)\left(D_{x}^{t} D_{y}^{s} \bar{w}\right),
\end{gathered}
$$

where $c_{s t}=\left(\begin{array}{c}l-1 \\ s\end{array}\right)\left(\begin{array}{c}n \\ t\end{array}\right), 0 \leq s \leq l-1$ and $0 \leq t \leq n$. If we freeze equation (4.1) at 0 , the right hand side will be zero since $t+s \leq n+l-1$ and so $D_{x}^{t} D_{y}^{s} w(0)=0$. In the left hand side of (4.1), the terms $D_{x}^{t+1} D_{y}^{s} w(0)$ in the sum are all equal to 0 except possibly when $t=n$ and $s=l-1$. But this latter term is multiplied by $b(0)=0$ and so we are led to the contradiction $D_{x}^{n} D_{y}^{l} w(0)=0$. This proves the claim.

Suppose now $D^{\alpha} h(0)=0$ for $|\alpha| \leq m-1$ so that near $0,|h(x, y)| \leq c(|x|+|y|)^{m}$ for some $c>0$. If $D^{\beta} w(0) \neq 0$ for some $|\beta| \leq m-1$, the preceding claim tells us that $D_{x}^{k} w(0) \neq 0$ for some $k \leq m-1$ and that $D_{x}^{n} D_{y}^{l} w(0)=0$ whenever $n+l \leq k$ and $l>0$. It follows that there are constants $c_{1}, c_{2}>0$ such that

$$
|w(x, y)| \geq c_{1}|x|^{k} \quad \text { if } \quad(x, y) \in \Gamma=\left\{(s, t):|t| \leq c_{2}|s| \text { and }|s|+|t| \leq c_{2}\right\} .
$$

Thus in $\Gamma$,

$$
\left|e^{-g}\right|=\frac{|w|}{|h|} \geq \frac{c_{2}|x|^{k}}{c(|x|+|y|)^{m}} \geq c_{3}|x|^{k-m}
$$

for some $c_{3}>0$. Since $k-m \leq-1$, the latter inequality tells us that $e^{-g} \notin L^{2}(\Gamma)$ which is a contradiction. Hence $D^{\alpha} w(0)=0$ for $|\alpha| \leq m-1$. The same reasoning also tells us that

$$
D^{\beta} w(0)=0 \text { for }|\beta| \leq m-1 \Longrightarrow\left|D^{\alpha} h(0)\right|=0 \text { for }|\alpha| \leq m-1 .
$$

Indeed, the claim is also valid for $h$ since it satisfies an equation of the type that $w$ satisfies with $A \equiv 0$ and $B \equiv 0$.

Corollary 4.6. Suppose $w, h$ and $g$ are as in Corollary 4.4 and that $w(0)=0=$ $h(0)$. Then given $\lambda>0$, there is a neighborhood $V_{\lambda}$ of 0 and $M_{\lambda}>0$ such that

$$
\left|e^{g(x, y)}\right| \leq M_{\lambda} \quad \text { and } \quad\left|e^{-g(x, y)}\right| \leq M_{\lambda} \quad \text { on } \Gamma=\left\{(s, t) \in V_{\lambda}:|t| \leq \lambda|s|\right\} .
$$


Proof. This follows from the proof of Corollary 4.4 (b). Indeed, for some $k \in \mathbb{N}$ and $c_{1}, c_{2}>0$ we saw that

$$
c_{1}|x|^{k}-c_{2}(|x|+|y|)^{k+1} \leq|w(x, y)| \leq c_{1}|x|^{k}+c_{2}(|x|+|y|)^{k+1}
$$

in a neighborhood of 0 . Now $h$ also satisfies similar inequalities with the same $k$.

We continue to assume that $L$ is hypoelliptic, $A$ and $B$ are smooth, $L w=$ $A w+B \bar{w}, L h=0$ and $e^{g}=\frac{h}{w}$, everything holding in a neighborhood of the closure of a ball $Q$. We assume that $0 \in Q$ and that it is the only zero of $h$ and $w$ in $Q$. The solvability in $B M O$ (in the $x$-variable) with arbitrary small norm in a sufficiently small neighborhood of 0 and the hypoellipticity of $L$ have the following consequence: given $p>1, e^{|g|} \in L^{p}(Q)$. By the hypoellipticity of $L$ we may also assume that we are in coordinates $(x, y)$ where $\mathcal{Z}(x, y)=x+i \phi(x, y)$ is a one-to-one first integral of $L, \phi$ real valued, and

$$
\phi(0)=0=\phi_{x}(0) .
$$

Modulo a nonvanishing factor, we then have

$$
L=\frac{\partial}{\partial y}+b(x, y) \frac{\partial}{\partial x}
$$

where

$$
b(x, y)=\frac{-i \phi_{y}}{1+i \phi_{x}} .
$$

In the next three propositions we will make the following additional assumption: the function $\phi(0, y)$ has a zero of finite order $N$ at 0 . This is equivalent to assuming that $L$ is of finite type, which occurs for example when $L$ is real analytic. We now recall from the proof of Corollary 4.4 that the functions $h(x, 0)$ and $w(x, 0)$ have a zero of the same order at 0 . Therefore, by the Malgrange Preparation Theorem we may write

$$
\begin{aligned}
& h(x, y)=q_{1}(x, y)\left(x^{k}+a_{k-1}(y) x^{k-1}+\cdots+a_{0}(y)\right), \\
& w(x, y)=q_{2}(x, y)\left(x^{k}+b_{k-1}(y) x^{k-1}+\cdots+b_{0}(y)\right),
\end{aligned}
$$

where $a_{j}(0)=0=b_{j}(0) \forall j$ and the $q_{i}$ are nonvanishing. Since $L$ is hypocomplex, (4.5) implies that $h(x, y)=\mathcal{Z}(x, y)^{k} f(x, y)$ for some nonvanishing $f$, where $\mathcal{Z}$ is the first integral we mentioned above. The latter together with (4.2) and the order assumption on $\phi(0, y)$ imply that there exist positive constants $A_{1}$ and $A_{2}$ such that

$$
A_{1}\left(|x|^{k}+|y|^{k N}\right) \leq|h(x, y)| \leq A_{2}\left(|x|^{k}+|y|^{k N}\right) .
$$

Proposition 4.7. $e^{-g}=\frac{w}{h}$ is bounded.

Proof. We claim that for some constants $B_{j}(j=0,1, \ldots, k-1)$,

$$
\left|b_{j}(y)\right| \leq B_{j}|y|^{(k-j) N} .
$$

Suppose the claim is not valid. Consider the finite set of integers

$$
S=\left\{j N+\operatorname{order} b_{j}(y): 0 \leq j \leq k-1\right\},
$$


and choose $\ell$ such that $\ell N+$ order $b_{\ell}(y)=$ the minimum of $S$, and $\ell$ is the smallest such. (Here order $b_{j}(y)$ refers to the order at 0 .) Since the claim is not valid, for $\epsilon$ small enough, in the region

$$
R_{\epsilon}=\left\{(x, y): \epsilon y^{N} \leq x \leq 2 \epsilon y^{N}, 0 \leq y \leq \epsilon\right\},
$$

we have

$$
|w(x, y)| \geq M_{1}\left|b_{\ell}(y) x^{\ell}\right| \geq M_{2} \epsilon^{\ell}|y|^{k N-1},
$$

for some positive constants $M_{1}$ and $M_{2}$. The latter together with (4.6) tell us that $e^{-g} \notin L^{N+1}\left(R_{\epsilon}\right)$, contrary to our choice of the neighborhood $Q$. Hence the claim is valid. By considering separately the regions $|x| \geq|y|^{N}$ and $|x| \leq|y|^{N}$, it follows from the claim that for some constant $A_{3}$,

$$
|w(x, y)| \leq A_{3}\left(|x|^{k}+|y|^{k N}\right) .
$$

The proposition now follows from (4.6) and (4.8).

The next proposition improves (4.7).

Proposition 4.8. For some constants $C_{j}(j=0,1, \ldots, k-1)$, we have

$$
\left|b_{j}(y)\right| \geq C_{j}|y|^{(k-j) N} .
$$

Proof. We first prove (4.9) for $j=0$. From (4.8) we know that $\left|b_{0}(y)\right| \leq M|y|^{k N}$ for some constant $M$. If $\left|b_{0}(y)\right| \leq \widetilde{M}|y|^{k N+1}$ for some $\widetilde{M}$, then by (4.7), in the region

$$
\mathcal{R}=\left\{(x, y): a y^{N+2} \leq x \leq 2 a y^{N+3}, 0 \leq y \leq a\right\}
$$

$|w(x, y)| \leq c|y|^{k N+1}$ for some $c>0$ and some small $a$. But then the latter together with (4.6) would imply that in $\mathcal{R}$,

$$
\left|\frac{h}{w}\right| \geq \frac{c_{1}}{|y|} \text { for } c_{1}>0
$$

contradicting the integrability to any power of $\frac{h}{w}$. Hence (4.9) holds for $j=$ 0 . Suppose now (4.9) holds for $j \leq m$. We wish to show that $\left|b_{m+1}(y)\right| \geq$ $C_{m+1}|y|^{(k-m-1) N}$ for some $C_{m+1}>0$. First observe that

$$
\left(\frac{\partial}{\partial x}\right)^{m}\left(\frac{\partial}{\partial y}\right)^{N(k-m)}(w)(0) \neq 0 .
$$

To see this, note that,

$$
\left(\frac{\partial}{\partial x}\right)^{m}\left(\frac{\partial}{\partial y}\right)^{N(k-m)}(w)(0)=\sum c_{s t}\left(\frac{\partial}{\partial x}\right)^{s}\left(\frac{\partial}{\partial y}\right)^{t} q_{2}(0)\left(\frac{\partial}{\partial x}\right)^{s^{\prime}}\left(\frac{\partial}{\partial y}\right)^{t^{\prime}} p(0),
$$

where the summation is taken over $\left\{s+s^{\prime}=m\right.$ and $\left.t+t^{\prime}=N(k-m)\right\}, p(x, y)=$ $x^{k}+b_{k-1}(y) x^{k-1}+\cdots+b_{0}(y)$ and $c_{s t}$ are nonzero integers. In the sum on the right, consider a term where $s^{\prime}<m$ :

$$
\left(\frac{\partial}{\partial x}\right)^{s^{\prime}}\left(\frac{\partial}{\partial y}\right)^{t^{\prime}} p(0)=\left(s^{\prime}\right) !\left(\frac{\partial}{\partial y}\right)^{t^{\prime}} b_{s^{\prime}}(0) \text {. }
$$


The induction assumption and (4.7) tell us that $b_{s^{\prime}}(y)$ is comparable to $|y|^{N\left(k-s^{\prime}\right)}$ and so $\left(\frac{\partial}{\partial y}\right)^{t^{\prime}} b_{s^{\prime}}(0)=0$. It follows that $\left(\frac{\partial}{\partial x}\right)^{m}\left(\frac{\partial}{\partial y}\right)^{N(k-m)} w(0)$ is a nonzero multiple of

$$
\left(\frac{\partial}{\partial x}\right)^{m}\left(\frac{\partial}{\partial y}\right)^{N(k-m)} p(0)=m !\left(\frac{\partial}{\partial y}\right)^{N(k-m)} b_{m}(0)
$$

and hence nonzero by the induction assumption.

Next apply $\left(\frac{\partial}{\partial x}\right)^{m}\left(\frac{\partial}{\partial y}\right)^{N(k-m)-1}$ to the equation

$$
\frac{\partial w}{\partial y}+b \frac{\partial w}{\partial x}=A w+B \bar{w}
$$

and evaluate at 0 . Since $\left(\frac{\partial}{\partial x}\right)^{j}\left(\frac{\partial}{\partial y}\right)^{\ell} w(0)=0$ for $j \leq m$ and $\ell \leq N(k-m)-1$ and that $\left(\frac{\partial}{\partial x}\right)^{m}\left(\frac{\partial}{\partial y}\right)^{N(k-m)} w(0) \neq 0$, we conclude: there is $s$ for which

$$
\left(\frac{\partial}{\partial y}\right)^{s} b(0)\left(\frac{\partial}{\partial x}\right)^{m+1}\left(\frac{\partial}{\partial y}\right)^{N(k-m)-1-s} w(0) \neq 0 .
$$

From (4.4) we know that the order of $b(0, y)$ at 0 is $N-1$ and so $s \geq N-1$ which implies that $N(k-m)-1-s \leq N(k-m-1)$. We thus have (4.9) for $j=m+1$ and the proposition is proved.

Proposition 4.9. $e^{g}=\frac{h}{w}$ is bounded.

Proof. Propositions 4.7 and 4.8 allow us to write

$$
w(x, y)=q_{2}(x, y)\left(x^{k}+c_{k-1} y^{N} x^{k-1}+\cdots+c_{1} y^{(k-1) N} x+c_{0} y^{k N}\right)+E(x, y),
$$

where each $c_{j} \neq 0$ and $|E(x, y)| \leq \alpha|y|\left(|x|^{k}+|y|^{k N}\right)$ for some $\alpha>0$. Let $|y| \leq \delta$ in the ball $Q$. By shrinking $Q$ if necessary, we can choose $m, M>0$ such that

$$
\sum_{j=0}^{k-1} \frac{\left|c_{j}\right|}{M^{k-j}}+\alpha\left(1+\frac{1}{M^{k}}\right) \delta<\frac{1}{2}
$$

and

$$
m^{k}+\sum_{j=1}^{k-1}\left|c_{j}\right| m^{j}+\alpha \delta\left(m^{k}+1\right)<\frac{\left|c_{0}\right|}{2} .
$$

It follows that in the region $\left\{(x, y) \in Q:|x| \geq M|y|^{N}\right\}$, we have

$$
\left|\frac{w(x, y)}{q_{2}(x, y)}\right| \geq \frac{1}{2}|x|^{k}
$$

while in the region $\left\{(x, y) \in Q:|x| \leq m|y|^{N}\right\}$,

$$
\left|\frac{w(x, y)}{q_{2}(x, y)}\right| \geq \frac{\left|c_{0}\right|}{2}|y|^{k N} .
$$

We therefore need only estimate $w(x, y)$ on the set

$$
\begin{aligned}
T & =\left\{(x, y) \in Q: m|y|^{N} \leq|x| \leq M|y|^{N}\right\} \\
& =\left\{\left(\tau y^{N}, y\right): m \leq \tau \leq M, \quad|y| \leq \delta\right\} .
\end{aligned}
$$


Let $f(\tau)=\tau^{k}+c_{k-1} \tau^{k-1}+\cdots+c_{0}$ and let $\frac{1}{\left|q_{2}(x, y)\right|} \leq c$ on $Q$. To finish the proof, in view of (4.6), it suffices to show that $f(\tau) \neq 0$ when $m \leq|\tau| \leq M$. Suppose $f\left(\tau_{1}\right)=0$ in the latter set, and write $|f(\tau)|=\left|\tau-\tau_{1}\right|^{r} q(\tau)$, where $q\left(\tau_{1}\right)>0$. Using $\left|w\left(\tau y^{N}, y\right)\right| \leq c|y|^{k N}\left(|f(\tau)|+|y|\left(|\tau|^{k}+1\right)\right)$ and (4.6), we have

$$
\left|\frac{h\left(\tau y^{N}, y\right)}{w\left(\tau y^{N}, y\right)}\right| \geq \frac{A_{2}}{c}\left(\frac{|\tau|^{k}+1}{\left|\tau-\tau_{1}\right|^{r} q(\tau)+|y|\left(|\tau|^{k}+1\right)}\right) .
$$

Choose $\tau_{2}>\tau_{1}$ such that $q(\tau)>0$ on $\left[\tau_{1}, \tau_{2}\right]$ and $\left|\tau_{2}-\tau_{1}\right|<1$. Then $\exists A_{3}>0$ such that for $\tau \in\left[\tau_{1}, \tau_{2}\right]$,

$$
\left|\frac{h\left(\tau y^{N}, y\right)}{w\left(\tau y^{N}, y\right)}\right| \geq \frac{A_{3}}{\left|\tau-\tau_{1}\right|+|y|} .
$$

Hence

$$
\begin{aligned}
\iint_{T}\left|\frac{h(x, y)}{w(x, y)}\right|^{N+2} d x d y & =\int_{0}^{\delta} \int_{m}^{M}\left|\frac{h\left(\tau y^{N}, y\right)}{w\left(\tau y^{N}, y\right)}\right|^{N+2}|y|^{N} d \tau d y \\
& \geq A_{3}^{N+2} \int_{0}^{\delta} \int_{\tau_{1}}^{\tau_{2}}\left(\frac{1}{\tau-\tau_{1}+|y|}\right)^{N+2}|y|^{N} d \tau d y \\
& =\infty
\end{aligned}
$$

contradicting the integrability of $\left(\frac{h}{w}\right)^{N+2}$. Thus $f(\tau) \neq 0$, and the proposition is proved.

\section{REFERENCES}

1. J. Alvarez and J. Hounie, Estimates for the kernel and continuity properties of pseudodifferential operators. Arkiv för Mat. 28 (1990), 1-22.

2. L. Bers, An outline of the theory of pseudoanalytic functions. Bull. Amer. Math. Soc. 62 (1956), 291-331. MR 18:470d

3. L. Bers, Theory of pseudo-analytic functions. Institute for Math. and Mech., New York Univ., New York, (1953). MR 15:211c

4. L. Bers and L. Nirenberg, On a representation theorem for linear elliptic systems with discontinuous coefficients and its applications. Convegno Internationale sulle Equazioni Lineari alle Derivate Parziali, Edition Cremonese(Rome), (1955), 111-140. MR 17:974d

5. D. Colton, Analytic theory of partial differential equations. Pitman(London), (1980). MR 82j:35001

6. D. Goldberg, A local version of real Hardy spaces. Duke Math. J. 46 (1979), 27-42. MR 80h:46052

7. J. Hounie, Global Cauchy problems modulo flat functions. Advances in Math. 51 (1984), 240-252. MR 86c:35158

8. J. Hounie and E. Perdigão, On local solvability in $L^{p}$ of first-order linear operators. J. of Math. Ann. Appl. 197 (1996), 42-53. MR 96j:35005

9. A. Meziani, On the similarity principle for planar vector fields. Application to Second Order PDE. J. Diff. Equations 157 (1999), 1-19. CMP 2000:01

10. L. Nirenberg and F. Treves, Solvability of a first order linear partial differential equation. Comm. Pure Appl. Math. 16 (1963), 331-351. MR 29:348

11. H. Renelt, Elliptic systems and quasiconformal mappings. Pure and Applied Mathematics, Wiley-Interscience, (1988). MR 90k:35086

12. E. M. Stein, Singular integrals and differentiability properties of functions. Princeton Univ. Press, (1970). MR 44:7280

13. E. M. Stein, Harmonic Analysis: Real-variable methods, orthogonality, and oscillatory integrals. Princeton Univ. Press, (1993). MR 95c:42002

14. E. M. Stein and G. Weiss, On the theory of harmonic functions of several variables I. Acta Math. 103 (1960), 25-62. MR 22:12315 
15. F. Treves, Hypoanalytic Structures: Local Theory. Princeton Univ. Press, (1992). MR 94e:35014

16. I. V. Vekua, Generalized Analytic Functions, Pergamon Press, Oxford, (1962). MR 27:321

Department of Mathematics, Temple University, Philadelphia, Pennsylvania 191226094

E-mail address: berhanu@euclid.math.temple.edu

Departamento de Matemática, Universidade Federal de São Carlos, Caixa Postal 676, CEP 13.565-905, SÃo Carlos, SP, Brasil

E-mail address: hounie@dm.ufscar.br

Department of Mathematics, Temple University, Philadelphia, Pennsylvania 19122 6094

Current address: Departamento de Matemática, Universidade Federal de Pernambuco, CEP 50.740-540, Recife, PE, Brasil

E-mail address: santiago@dmat.ufpe.br 